\title{
Pharmacokinetics of progesterone in lactating dairy cows: Gaining some insights into the metabolism from kinetic modeling
}

\author{
L. N. Turino, ${ }^{*}$ R. N. Mariano, ${ }^{*}$ M. I. Cabrera, ${ }^{*}$ D. E. Scándolo,† M. G. Maciel,† and R. J. A. Grau**1 \\ *Laboratorio de Química Fina, Instituto de Desarrollo Tecnológico para la Industria Química (INTEC), Universidad Nacional del Litoral, \\ Consejo Nacional de Investigaciones Científicas y Técnicas (CONICET), Predio CCT-CONICET Santa Fe, 3000 Santa Fe, Argentina \\ †Instituto Nacional de Tecnología Agropecuaria (INTA), Estación Experimental Agropecuaria Rafaela, 2300 Rafaela, Argentina
}

\begin{abstract}
Progesterone pharmacokinetics were analyzed for plasma hormone concentrations ranging from linear to saturated metabolism in lactating Holstein cows with differing daily milk yields. The adequacy of 2-coupled first-order (bi-exponential equation), hyperbolic (Michaelis-Menten equation), and sigmoidal (Hill equation) kinetic models to describe the experimental progesterone pharmacokinetic profiles was examined on a statistical basis. After nonlinear regression and statistical analysis of the data-fitting capability, a simple one-compartment model based on Hill equation proved to be most adequate. This model indicates an enzyme-catalyzed metabolism of progesterone involving cooperative substrate-binding sites, resulting from allosteric effects that yield a sigmoidal saturation rate curve. Kinetic parameters were estimated for 2 groups of lactating Holstein cows with different daily milk yields. We found, for the first time, a remarkable quantitative agreement of the Hill coefficient value with that reported in pharmacokinetic studies involving cytochrome $\mathrm{P} 450$, family 3, subfamily A (CYP3A)-mediated reactions in other mammals, humans included. It seems that positive cooperativity makes enzymes much more sensitive to plasma progesterone concentration, and their activities can undergo significant changes in a narrow range of concentration as characteristic of sigmoidal behavior. Therefore, the values of classical pharmacokinetic parameters, such as the elimination constant, half-life, and clearance rate, were found to be highly dependent on the plasma progesterone concentration.
\end{abstract}

Key words: dairy cow, progesterone metabolism, Hill equation, pharmacokinetic parameters

\section{INTRODUCTION}

Progesterone plays a prominent role in drug intervention programs to control the estrous cycle of cattle because it inhibits the expression of estrus and prevents

Received June 27, 2009.

Accepted November 14, 2009.

${ }^{1}$ Corresponding author: cqfina@santafe-conicet.gov.ar ovulation by suppressing LH release (Shrestha et al., 2004; Colazo et al., 2008). Since the early 1960s, considerable efforts have been made to gain a better understanding of the biochemical and physiological principles controlling reproductive processes as well as circulating progesterone concentrations (Miller et al., 1963; Gomes and Erb, 1965; Butler, 2003). Plasma progesterone concentration is governed by dosing regimens and a variety of physiological mechanisms including plasma protein binding, accumulation in adipose tissue, absorption in tissues and organs containing progesterone receptors, and excretion (Williams, 1962; Hamudikuwanda et al., 1996; Rabiee et al., 2001a). Major efforts on the use of progesterone for parenteral and intravaginal administration in dairy cattle reproductive management programs have been devoted to study the dose effects. The efficacy of the administered progesterone, however, is known to be related to the hormone concentration in the bloodstream.

Despite great concern about the need to use reliable frameworks to describe progesterone pharmacokinetics in dairy cattle, only empirical function-based pharmacokinetic models have been used for this purpose. Broadly applicable mechanistic pharmacokinetic models are scarce or absent. Indeed, usual approaches quantify simple pharmacokinetic parameters such as progesterone metabolic clearance rates from continuous infusion procedures and from the residual progesterone content in used intravaginal inserts (Rabiee et al., 2001b, 2002; Sangsritavong et al., 2002). Both approaches are performed without the need to use pharmacokinetic models or sophistications such as multi-compartmental analysis. Estimates from these methods, however, are certainly limited in pharmacokinetic information compared with that provided by pharmacokinetic models. Simple 2-compartment models have been proposed to describe progesterone disposition kinetics, for instance, in dromedary camels (Al-Busadah and Homeida, 2004) and rhesus monkeys (Anand Kumar et al., 1982). Because this approach assumes a linear pharmacokinetic pattern, analytical solutions are sums of exponential terms, which allow further simplifications and avoid us- 
ing numerical integration algorithms. In general, these advantageous features promote the use of these types of mathematical models in pharmacokinetic studies but they neither provide a good data fitting at high plasma drug concentrations nor give insights into the catabolic mechanism involved. Conversely, nonlinear models are not easy to be solved, but they could be used as a tool to elucidate the underlying mechanisms and to describe nonlinearities attributable to cooperative or pharmacokinetic saturation effects. These latter features encouraged us to investigate the capability of nonlinear models for describing the intrinsic pharmacokinetics of progesterone in dairy cattle.

In the present contribution, the progesterone pharmacokinetic behavior is analyzed on plasma hormone concentrations ranging from linear to saturated metabolism in lactating Holstein cows with differing daily milk yields. The adequacy of linear 2-compartment kinetics (bi-exponential decay), nonlinear (saturation) kinetics (Michaelis-Menten equation), and nonlinear (cooperative) kinetics (Hill equation) to describe the experimental progesterone pharmacokinetic profiles is examined statistically. Based on the underlying mechanism supporting the pharmacokinetic model that better fits the experimental data, a mechanistic basis primarily responsible for the sigmoidal observed pharmacokinetic behavior is suggested. Kinetic parameters are estimated for 2 groups of lactating Holstein cows with different daily milk yields. The performance of pharmacokinetic parameters, such as the elimination constant, elimination half-life, and clearance rate, is analyzed. Some insights into the metabolism are discussed from a kinetic modeling viewpoint.

\section{MATERIALS AND METHODS}

\section{Animals and General Procedures}

Lactating Holstein cows from Experimental Station INTA Rafaela (Santa Fe, Argentina) at different weeks postpartum were milked twice a day at 0500 and 1500 $\mathrm{h}$ and fed ad libitum. Nonovariectomized animals were chosen because the liver enzymes involved in steroid metabolism may decrease in the absence of steroids (Freetly and Ferrell, 1994; Sangsritavong et al., 2002). Cows were weighed, scored for body condition (1 = lean, 5 = fat), and evaluated for milk production for $7 \mathrm{~d}$ before the start of the assay. To avoid endogenous progesterone production, ovarian function was monitored using a veterinary ultrasound scanner (Aquila, Pie Medical, the Netherlands). If a corpus luteum was found, cows were treated with 2 doses of $2 \mathrm{~mL}$ of prostaglandin [sodic $\mathrm{D}(+)$ cloprostenol, $75 \mu \mathrm{g} / \mathrm{mL}$, lot 63528; Aviar S.A. Laboratories, Buenos Aires, Ar- gentina] at intervals of $12 \mathrm{~h}$; these cows were used for experiments within $24 \mathrm{~h}$ after the last injection. If follicles were found, the cows were tested in experiments within $24 \mathrm{~h}$ after ultrasound monitoring. Cows were specifically excluded from the study if they had a basal progesterone concentration $>1 \mathrm{ng} / \mathrm{mL}$ in a pretreatment blood sample.

\section{Materials}

Progesterone (99.2\%, Sigma-Aldrich, Buenos Aires, Argentina) was US Pharmacopeia grade. Benzyl alcohol (99\%, Cicarelli, Buenos Aires, Argentina), 2-pyrrolidone (99\%, Sigma-Aldrich), and physiological saline $(0.9 \% \mathrm{wt} / \mathrm{vol}$ sodium chloride) were filtered through cellulose acetate syringe filters $(0.22 \mu \mathrm{m})$ before use. A sterile injectable solution of progesterone $(0.67 \% \mathrm{wt} /$ vol) was prepared under sterile conditions as follows: progesterone powder $(0.67 \mathrm{~g})$ was added to a solution of benzyl alcohol $(4.00 \mathrm{~mL})$ and 2-pyrrolidone $(56.00 \mathrm{~mL})$ and stirred until completely dissolved at room temperature. Then, this solution was diluted with physiological saline $(40.00 \mathrm{~mL})$.

\section{Pharmacokinetic Assays}

The study was conducted in 10 lactating Holstein cows, which were sorted into 2 groups according to days postpartum and milk production, as summarized in Table 1 . The sterile solution of progesterone $(15 \mathrm{~mL}, 100$ $\mathrm{mg}$ of progesterone) was injected into the jugular vein of each cow. Serial blood samples for pharmacokinetic modeling were collected from each animal at 1, 3, 6, 10, 15, 30, 60, and $90 \mathrm{~min}$ and at 2, 3, and $6 \mathrm{~h}$ after injection. Blood samples were collected from the coccygeal vessel into tubes containing $0.07 \mathrm{~mL}$ of EDTA solution (0.342 mol/L, pH 7.2, Wiener, Rosario, Argentina) and processed to yield plasma. After centrifugation of blood at 3,000 $\times g$ for $10 \mathrm{~min}$, plasma samples were stored at $-20^{\circ} \mathrm{C}$ until further analysis.

\section{Hormone Analysis}

Progesterone plasma concentrations were determined by radioimmunoassay using a commercial solid-phase, I kit (Coat-A-Count kit, Siemens Medical Solutions Diagnostics, Los Angeles, CA). Duplicate analyses were performed on each sample. The intraassay coefficient of variation was $<7 \%$ for concentrations between 0.1 and $40.0 \mathrm{ng} / \mathrm{mL}$, the interassay coefficient of variation was $3.5 \%$, and the sensitivity was $0.01 \mathrm{ng} / \mathrm{mL}$.

\section{Candidate Kinetic Models}

Special care was taken to eliminate the most significant endogenous source of progesterone (regression of 
Table 1. Postpartum days, milk production, BW, and BCS of the cows of each group (group A = highproducing; group B = low-producing)

\begin{tabular}{llcccc}
\hline Group & Cow no. & Days postpartum & Milk production $(\mathrm{L} / \mathrm{d})$ & BW $(\mathrm{kg})$ & BCS \\
\hline A $(\mathrm{n}=5)$ & 3369 & 38 & 41.6 & 716 & 2.50 \\
& 4237 & 51 & 40.9 & 667 & 2.75 \\
& 4067 & 40 & 38.0 & 542 & 2.75 \\
& 4548 & 43 & 33.6 & 510 & 2.75 \\
& 3668 & 70 & 33.2 & 649 & 2.50 \\
& Average & $48^{\mathrm{a}}$ & $37.5^{\mathrm{a}}$ & $617^{\mathrm{b}}$ & $2.65^{\mathrm{b}}$ \\
& SD & 3.9 & 87.0 & 0.14 \\
& \% CV & 13.0 & 10.5 & 14.1 & 5.17 \\
$\mathrm{~B}(\mathrm{n}=5)$ & 26.9 & 23.8 & 530 & 2.75 \\
& 4450 & 150 & 22.5 & 643 & 2.25 \\
& 2896 & 151 & 20.7 & 577 & 2.75 \\
& 3690 & 490 & 14.8 & 798 & 3.25 \\
& 3177 & 540 & 19.6 & 662 & 2.50 \\
& 2454 & 436 & 4.3 & $642^{\mathrm{b}}$ & $2.70^{\mathrm{b}}$ \\
& Average & $353^{\mathrm{a}}$ & 22.4 & 102 & 0.37 \\
& SD & 189 & & 15.9 & 13.73 \\
\hline
\end{tabular}

${ }^{a}$ Means between groups were different $(P<0.05)$.

${ }^{\mathrm{b}}$ Means between groups were equal $(P>0.05)$.

the corpus luteum) and to avoid using long-time experimental data, which can be significantly disguised by the phenomenon of progesterone generation from the adrenal cortex. From data collected in pharmacokinetic assays, plasma progesterone concentrations ranged from basal levels up to $350 \mathrm{ng} / \mathrm{mL}$. Because the range of concentrations was so wide, we hypothesized that a linear pharmacokinetic model would not necessarily be expected. Accordingly, the kinetic modeling was performed without expressing an opinion a priori on whether the pharmacokinetics was linear or nonlinear. Indeed, the performance of the proposed models in also predicting linear kinetics arises naturally as a result of their asymptotic behaviors. Admitting that nonlinearities may be at different degrees of absorption, distribution, or elimination, rigorous modeling could be very demanding and lead to complex models. To avoid solving models with too many parameters and requiring too much data, we made an attempt to suggest the simplest model without loss of accuracy. The data were analyzed by using the following simplified candidate kinetic models.

Model 1: Two-Coupled First-Order Kinetics. Basic underlying assumptions are multi-compartment distribution and first-order kinetics to absorption/ distribution and elimination processes. Simply, it is assumed that progesterone elimination occurs from a central compartment (blood and well-perfused organs) linked to another peripheral (poorly perfused tissues), as illustrated in the scheme shown in Figure 1a. Thus, the progesterone metabolism rate equations can be written as

$$
\frac{\mathrm{d} C x}{\mathrm{~d} t}=k_{21} C y-k_{12} C x-k_{10} C x
$$

$$
\frac{\mathrm{d} C y}{\mathrm{~d} t}=k_{12} C x-k_{21} C y,
$$

in which $C x$ and $C y$ are the progesterone concentrations in the central $(x)$ and peripheral $(y)$ compartments, respectively; $k_{12}, k_{21}$, and $k_{10}$ are kinetic constants, and $t$ is the time after progesterone administration. The coupling between both compartments can be a source of nonlinearity. Indeed, this model describes a bi-exponential decay of the hormone, as is commonly found in fitting plasma concentration-time profiles of most drugs. If the dynamics of the progesterone into $y$ is much slower than into $x$, equations [1] and [2] approach a model describing a progesterone metabolism obeying first-order elimination kinetics, with quasi-steady state distribution into the compartment $y$. Parameters $k_{12}$, $k_{21}$ and $k_{10}$ remain to be determined.

Model 2: Hyperbolic Kinetics. Basic underlying assumptions are that the enzyme-substrate complex formation rate is much faster than the product-forming rate, capacity-limited metabolism, and noncompartment analysis, as depicted in the scheme in Figure 1b. The first assumption leads to the classical simplifying approximation of quasi-steady-state. The second assumption is in accordance with the fact that the saturation of enzymatic metabolism is one of the most common sources of nonlinearity (Mehvar, 2001). The third basic assumption implies that enzymatic metabolism is the unique pathway of elimination. This means rapid distribution of hormone to the major organs of biotransformation and elimination (e.g., liver and kidneys), which in turn have relatively high blood perfusion. After assuming Michaelis-Menten kinetics, the progesterone metabolism rate equation can be expressed as 


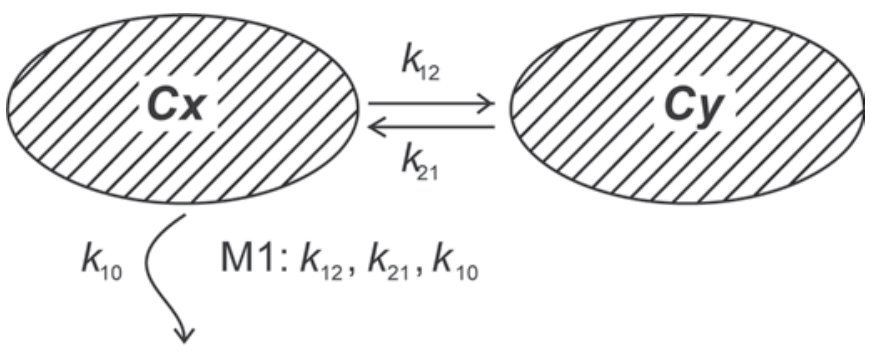

(a)

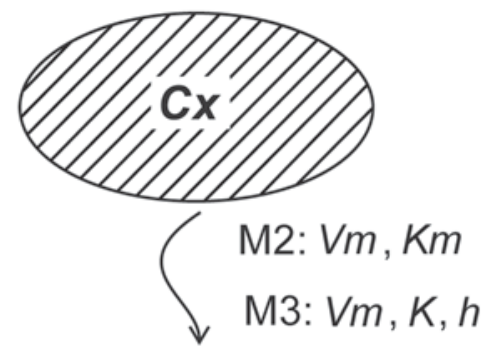

(b)

Figure 1. Schematic representation of (a) the bi-compartmental model 1 (M1; 2-coupled first-order kinetics); and (b) mono-compartmental models 2 (M2; hyperbolic kinetics) and 3 (M3; sigmoidal kinetics). $C x$ and $C y$ are the progesterone concentrations in the central $(x)$ and peripheral $(y)$ compartments, respectively; $k_{12}, k_{21}$, and $k_{10}$ are kinetic constants; $\mathrm{Vm}$ is the maximum rate of metabolism; $\mathrm{Km}$ is the concentration producing half of $V m ; K$ is a constant including for the interaction factors; and $h$ is the Hill coefficient.

$$
\frac{\mathrm{d} C x}{\mathrm{~d} t}=-\frac{1}{V x} \frac{V m C x}{K m+C x},
$$

in which $C x$ is the progesterone concentration in the single compartment $x ; V x$ is the distribution volume of the compartment where the elimination is produced (see Figure 1); $V m$ is the maximum rate of metabolism; and $K m$ is the concentration producing half of $V m$. At one extreme, a linear behavior could be described when the concentration of available enzymes is much larger than the number of hormone molecules. At the other extreme, no change in the metabolism rate is described because all of the enzymes are saturated with hormone molecules. Whereas at the 2 extremes the metabolism rate follows either first- or zero-order kinetics, an intermediate order can be observed at a drug concentration around $K m$. Independent of the model, the volume $V x$ can be estimated as

$$
V x=D / C o
$$

in which $D$ is the administered dose and $C o$ is the plasma progesterone concentration determined as the coordinate-intercept from linear regression on the concentration data in the initial linear range of the curve $C x(t)$. Therefore, only parameters $V m$ and $K m$ have to be determined by nonlinear regression.

Model 3: Sigmoidal Kinetics. Basic underlying assumptions are enzyme-substrate complexes at quasi-steady-state, capacity-limited metabolism, and one-compartment analysis for the same reasons stated in model 2. Moreover, the model hypothesized competitive inhibition or activation of the enzyme reaction. Therefore, the observed kinetics cannot be described by a simple Michaelis-Menten equation. Accordingly, the Hill equation for an $h$-binding site enzyme is proposed to describe positive $(h>1)$ or negative $(h<1)$ cooperative kinetic profiles:

$$
\frac{\mathrm{d} C x}{\mathrm{~d} t}=-\frac{1}{V x} \frac{V m C x^{h}}{K^{h}+C x^{h}},
$$

in which $K$ is a constant including for the interaction factors and no longer equals $K m$, and $h$ is the Hill coefficient. It is conspicuous that equation [5] reduces to equation [3] only if $h=1$. This model displays a sigmoidal rate saturation curve. Parameters $V m, K$, and $h$ remain to be determined.

\section{Model Parameter Estimation and Model Discrimination}

Kinetic parameter optimization was carried out fitting plasma progesterone concentration versus time from 96 observations. Nonlinear regression analysis of experimental data was directly performed, avoiding log-linearization of the nonlinear models to minimize error propagations. The residual sum of squares $(\boldsymbol{S S Q})$ between experimental data and predictions were minimized by a modified Levenberg-Marquardt algorithm combined with a procedure for solving nonlinear least squares problems (Marquardt, 1963). The numerical integration of the rate equations was performed using a Runge-Kutta (2,3) pair method (Brankin et al., 1991). The optimization of kinetic parameters was achieved fitting the experimental data of plasma progesterone concentration in the time domain for all 3 kinetic models. Different initial value sets resulted in the same optimal set of model parameter values. Analysis of variance and data analysis were done employing Statgraphics Plus 5.1 (StatPoint Technologies, Warrenton, VA). Results were considered statistically significant if $P<0.05$. Models with $P<0.05$ were considered valid 
models because 95 out of 100 model predictions are statistically significant. The analysis of the significance of the overall regression and goodness-of-fit were appraised based upon Fisher's test (Froment and Hosten, 1981; Froment and Bischoff, 1990), which for only one species reduces to the following equation:

$$
F \text { calc }=\frac{(N-p) \sum_{i=1}^{N}\left(\text { Ccalc }_{i}\right)^{2}}{p \sum_{i=1}^{N}\left(C x_{i}-C^{2} a l c_{i}\right)^{2}},
$$

in which $C x_{i}$ and $C c a l c_{i}$ are the experimental and calculated plasma progesterone concentrations of the $i$ th data point, respectively; $N$ represents the number of experimental data; and $p$ is the number of adjusted parameters of the model. The regression was considered to be meaningful when the Fcalc value was greater than the corresponding tabulated $F$-value. The highest Fcalc value was taken as indicative of the best possible regression. The adequacy of the fitting was also checked examining the residual plots.

\section{Pharmacokinetic Parameter Evaluation}

The elimination constant $(\boldsymbol{K e l})$, the elimination halflife $\left(\boldsymbol{t}_{1 / 2}\right)$, and clearance $(\boldsymbol{C l})$ were calculated according to the following relationships:

$$
\begin{gathered}
K e l=\frac{\partial C x}{\partial t} \frac{1}{C x}, \\
t_{1 / 2}=\frac{0.693}{K e l} \\
C l=\frac{\partial C x}{\partial t} \frac{V x}{C x} .
\end{gathered}
$$

The relationship between all parameters and milk production groups was assessed using ANOVA tests.

\section{RESULTS}

Figure 2 illustrates model simulations and experimental plasma progesterone concentration data as a function of time for the 3 kinetic models and for the highest (cow no. 3369) and lowest (cow no. 2454) milkproducing cows. A simple visual inspection reveals that model 2 is less suitable than models 1 and 3, especially for middle values of plasma progesterone concentra- (a)

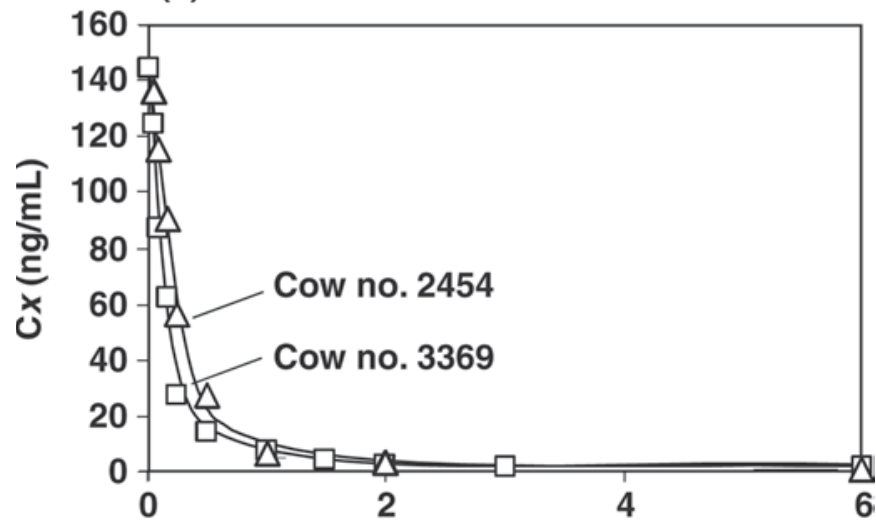

(b)

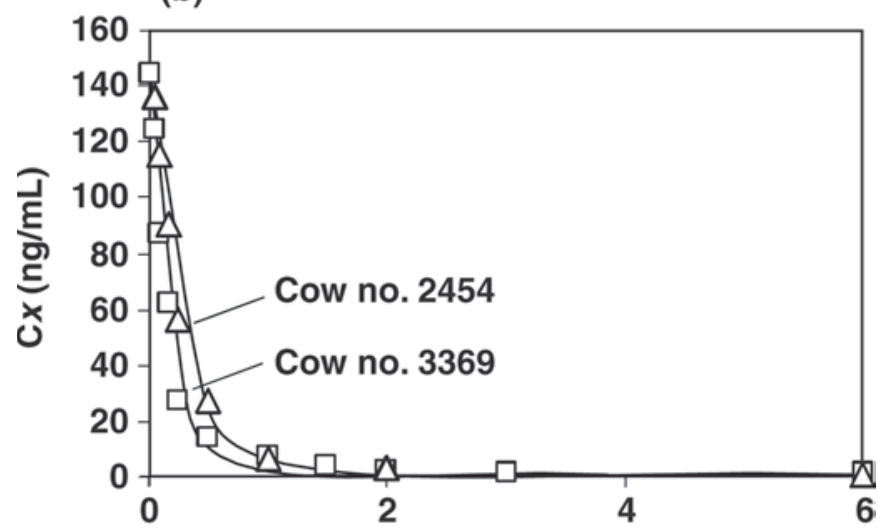

(c)

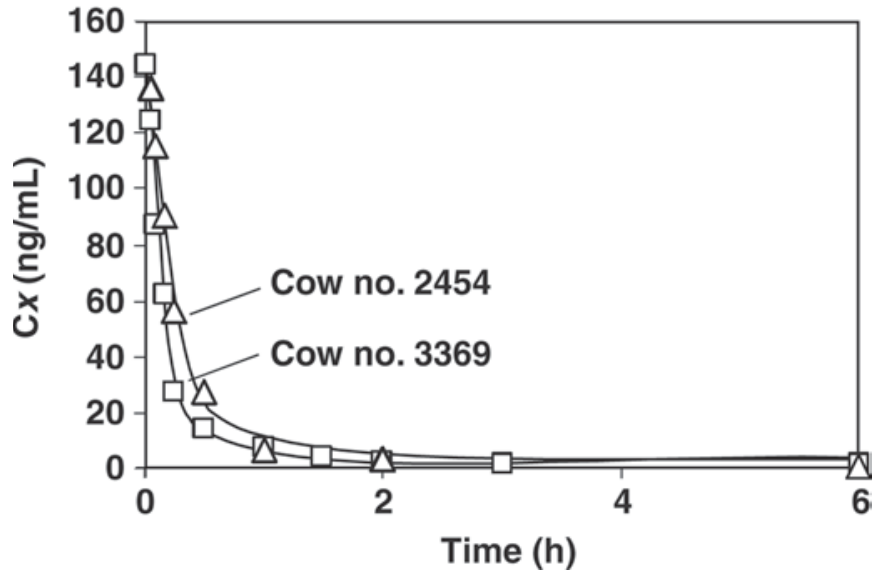

Figure 2. Model simulations and experimental plasma progesterone concentration $(C x)$ data as a function of time for the highest $(\square)$ and lowest $(\Delta)$ milk-producing cows: a) model 1 ; b) model 2; c) model 3 .

tions. Figure 3 shows the residuals between model predictions and experimental concentrations for the collection of data from all cows. The larger residual band for model 2 at high to middle concentrations reinforces the poor fitting model in comparison with models 1 and 3 . These latter 2 models display a similar scattering 
(a)

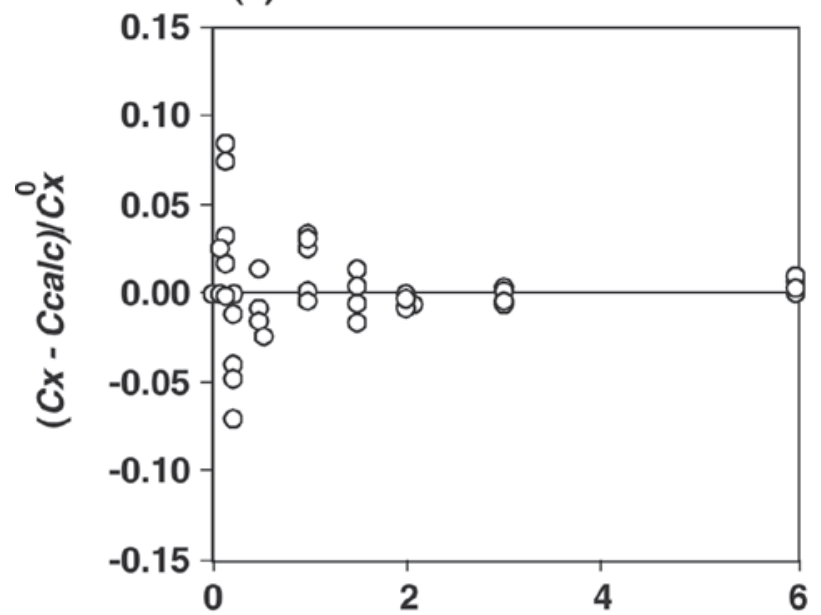

(c)

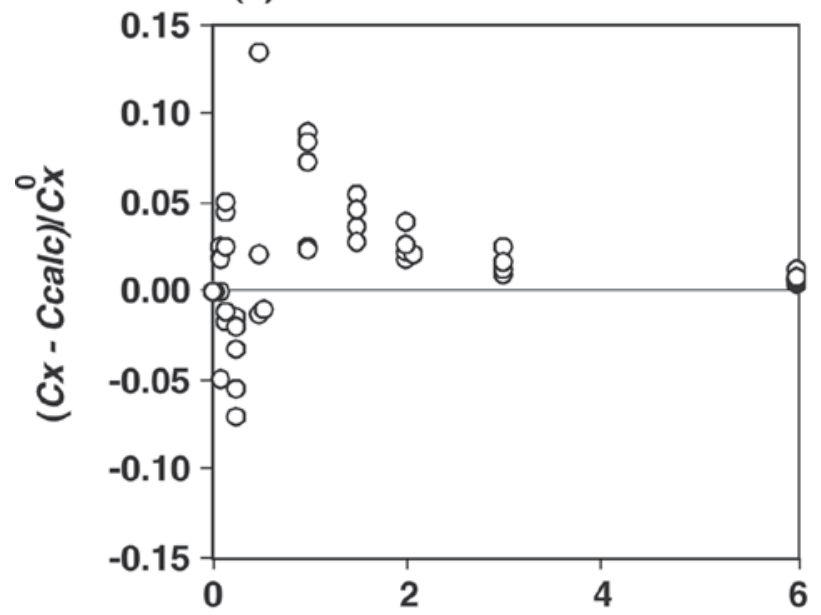

(e)

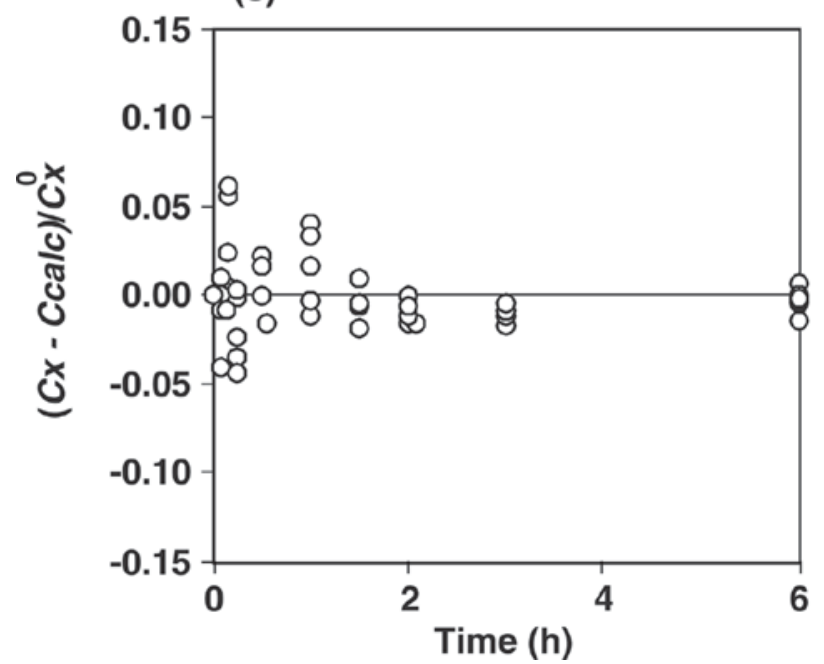

(b)

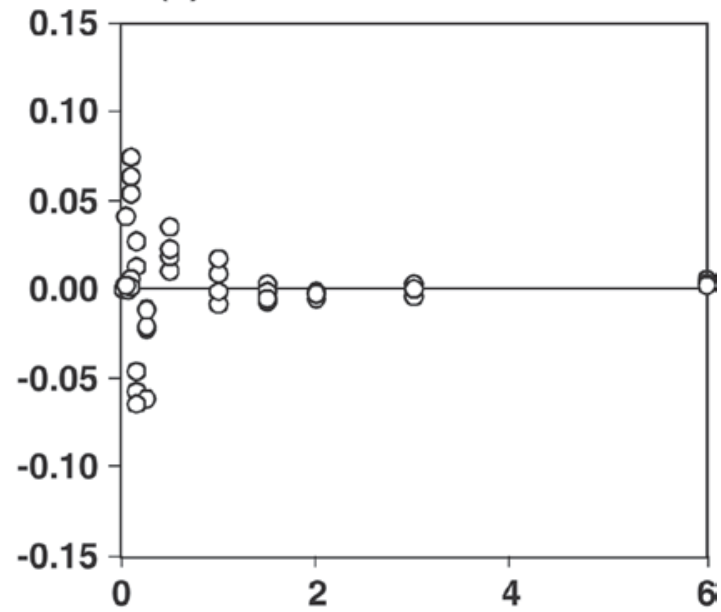

(d)

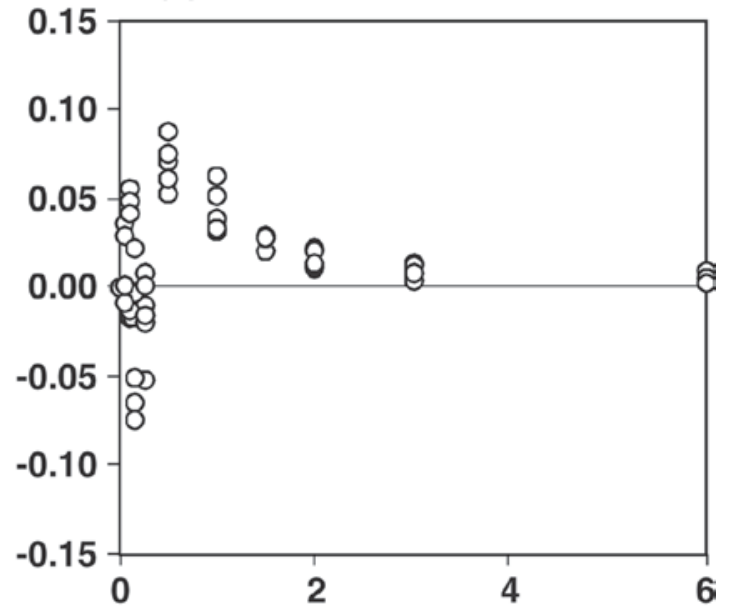

(f)

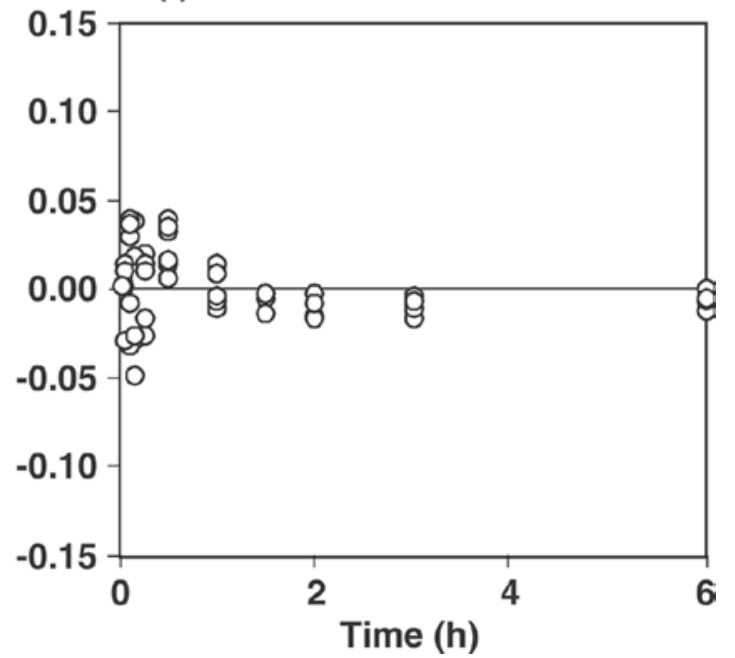

Figure 3. Residuals between model predictions (Ccalc) and experimental concentrations $(C x)$ for the collection of data from all cows: a) and b) = model 1 for A and B groups, respectively; c) and d) = model 2 for A and B groups, respectively; e) and f) = model 3 for A and B groups, respectively. 
Table 2. Values of the residual sum of squares $(S S Q)$ and Fisher's test (Fcalc) for the 3 rival kinetic models

\begin{tabular}{lcccr}
\hline Model & Parameters $(\mathrm{n})$ & $\mathrm{df}$ & SSQ & \multicolumn{1}{c}{ Fcalc } \\
\hline $1^{*}$ & 3 & 66 & $2,569.4$ & $8,139.1$ \\
$2^{*}$ & 2 & 76 & $4,813.5$ & $6,681.7$ \\
$3^{*}$ & 3 & 66 & $1,386.2$ & $15,391.2$ \\
\hline
\end{tabular}

*Statistically significant at $P<0.05$.

of the relative errors at the middle to lowest values of progesterone concentration. Consequently, by simple visual inspection, the discrimination between both rival models could be open to biased interpretation.

A better criterion for model discrimination is given by the statistical analysis of the data fitting capability. Table 2 summarizes the resulting $S S Q$ and Fcalc values for the 3 kinetic models. Results confirm that model 2 provides the worst quality in describing the kinetic behavior because it markedly exhibits the highest $S S Q$ value and the lowest Fcalc value. Thus, model 2 based on nonlinear Michaelis-Menten kinetics is ruled out from further consideration. Significant differences between models 1 and 3 now arise from the $S S Q$ and Fcalc values. Model 3, based on the Hill equation, is certainly favored, and it proved to be the best candidate to describe the experimental data set. Upon finding evidence that progesterone metabolism enzyme(s) basically follows the Hill equation, we drew plots of experimental and predicted progesterone metabolism rates as a function of the progesterone concentration. Figure 4 displays S-shaped curves as depicted by Hill plots for nonlinear interaction effects, such as allosteric/ cooperative binding $(h \neq 1)$. It can also be viewed that model 3 predictions fit to the experimental rate data quite well from unsaturated to saturated metabolism.

Model 3 mechanistically supports the possibility of an enzyme-catalyzed metabolism of progesterone involving cooperative substrate-binding sites. To gain more insight into the operating mechanism, values of the estimated model parameters and their physical meanings were examined. Table 3 summarizes the estimated parameters of model 3 for the 2 groups of cows. Concerning the estimates, no direct information is currently available for comparison purposes because some of them are reported for the first time in the present contribution; for example, parameters $K$ and $h$. An ANOVA test was performed to find out if there were any statistical differences between the parameter values characterizing the A and B groups of cows. No significant differences were found in the $K$ and $h$ variables between both groups $(P \gg 0.05)$, except for $V m(P \ll$ $0.05)$, as expected and argued in the Discussion section. In an attempt to check the accuracy of the model parameter values resulting from the multi-parametric curve-fitting procedure, $V m / V x$ ratio values were estimated, as is usually done, from the slope $(B=V m / V x)$ of the regression line of plasma progesterone concentrations on time periods in which the hormone is depurated to constant rate. Table 4 summarizes the results for comparison purposes. The good agreement between the values resulting from quite different approaches is indicative of an agreeable nonlinear parameter estimation and, having ruled out model 2, the evident linear kinetic behavior at short times motivates our preference for model 3 over model 1 . Moreover, the values of the Hill coefficient $h$ were found to be in close agreement with those reported in pharmacokinetic modeling studies involving other mammals, as reviewed below.

The basic pharmacokinetic parameters $\mathrm{Kel}, t_{1 / 2}$, and $\mathrm{Cl}$ displayed an unusual performance attributable to the sigmoidal kinetic behavior. In contrast with first-order kinetics, they are not constant values but functions depending on the plasma progesterone concentration $C x$, as settled by equations [7] to [9] coupled to equation [5]. Figure 5 shows such dependencies. The progesterone concentration or dose that yields maximum values of $\mathrm{Kel}$ and $\mathrm{Cl}$ (and minimum $\mathrm{t}_{1 / 2}$ ) is presented in Table 5. This progesterone concentration was found to be highly variable for each individual cow, even within same group (CV 36\% and $22 \%$ for groups A and B, respectively). Less variability was observed when using the progesterone dose producing the extreme values

Table 3. Pharmacokinetic parameter values [mean (SD)] of model 3 based on the Hill equation ${ }^{1}$

\begin{tabular}{lccccc}
\hline Group & $\begin{array}{c}V m / V x \\
(\mathrm{ng} / \mathrm{mL} \text { per } \min )\end{array}$ & $V x(\mathrm{~L})$ & $\begin{array}{c}V m(\mathrm{mg} / \mathrm{min} \\
\text { per kg) }\end{array}$ & $\begin{array}{c}K(\mathrm{ng} / \mathrm{mL} \\
\text { per } \mathrm{kg})\end{array}$ & $h$ (dimensionless) \\
\hline A $(\mathrm{n}=5)$ & 23.53 & 434 & $0.015^{\mathrm{a}}$ & $0.138^{\mathrm{b}}$ & $2.38^{\mathrm{b}}$ \\
& $(8.19)$ & $(135)$ & $(0.003)$ & $(0.041)$ & $(0.23)$ \\
B $(\mathrm{n}=5)$ & 9.98 & 537 & $0.008^{\mathrm{a}}$ & $0.115^{\mathrm{b}}$ & $2.08^{\mathrm{b}}$ \\
& $(1.49)$ & $(174)$ & $(0.001)$ & $(0.029)$ & $(0.41)$ \\
\hline
\end{tabular}

${ }^{a}$ Means between groups were different $(P<0.05)$.

${ }^{b}$ Means between groups were equal $(P>0.05)$.

${ }^{1} \mathrm{Vm}=$ maximum rate of metabolism; $V x=$ distribution volume; $K=$ constant including for the interaction factors; $h=$ Hill coefficient. 


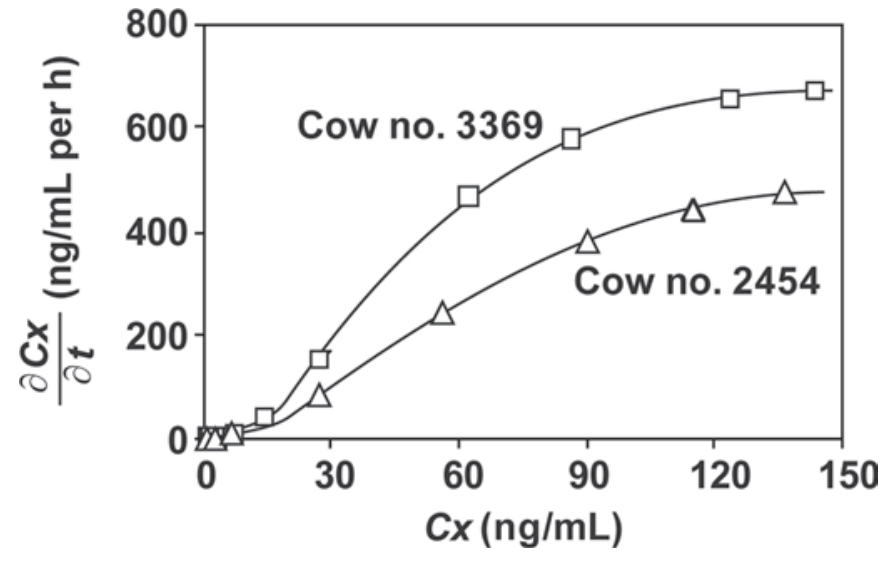

Figure 4. Experimental data and model 3 predictions of the progesterone metabolism rate $(\partial C x / \partial t)$ as a function of plasma progesterone concentration $(C x)$ for the highest $(\square)$ and lowest $(\Delta)$ milkproducing cows.

of these basic pharmacokinetic parameters (CV $23 \%$ and $20 \%$ for groups A and B, respectively). Significant differences were found between cows of different daily milk yields $(P \ll 0.05)$.

\section{DISCUSSION}

Inasmuch as the entire amount of the predetermined dose of progesterone was delivered directly into the bloodstream, a considerable and rapid serum protein binding of progesterone is most likely to occur because of the highly lipophilic nature of this small molecule $(314 \mathrm{Da})$. It is then expected that progesterone flowing through the bloodstream reaches, effectively and quickly, the liver, kidney, brain, ovary, and adrenal, which play a major role in the overall biotransformation and total body clearance of progesterone compared with those occurring in other organs and tissues (Bedford et al., 1974; Parr et al., 1993; Sangsritavong et al., 2002). Despite the previously mentioned diverse ways of metabolizing progesterone, our analysis shows the successful use of a simple one-compartment model to describe the pharmacokinetics within a concentration range sufficiently large to move from linear to saturated metabolism. As to the first conclusion, the enzyme(s)-mediated reaction is a rate-determining step in the present study; otherwise, it would be necessary to perform a multi-compartment analysis.

To formulate the kinetic model without a priori assumptions (independent of the observations) on saturation phenomena, the experimental design was intentionally addressed to include plasma progesterone concentrations much larger than those used in hormonal protocols for synchronization of ovulation and timed AI in dairy cows. The possibility of nonlinear (a)

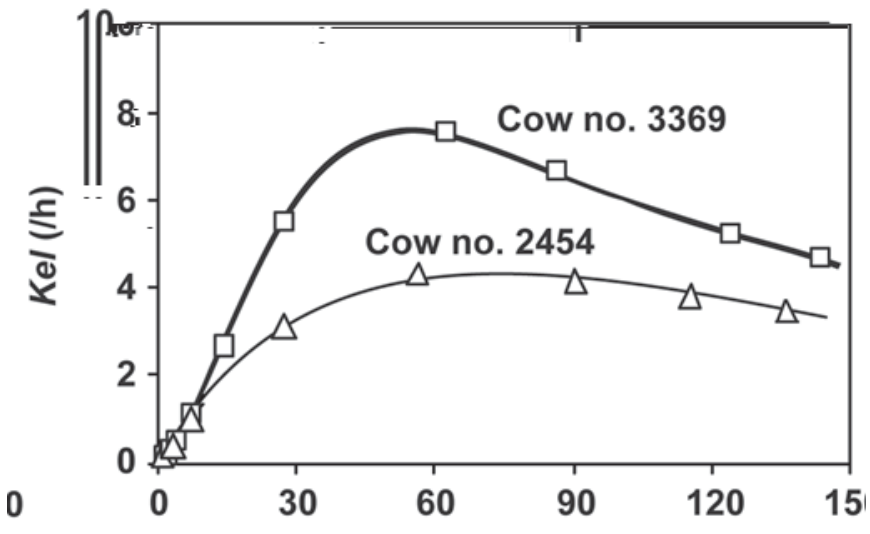

(b)

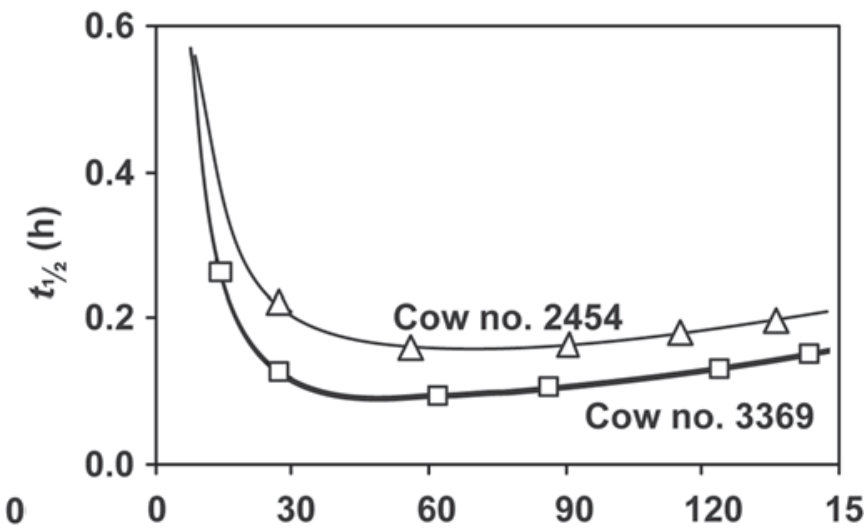

(c)

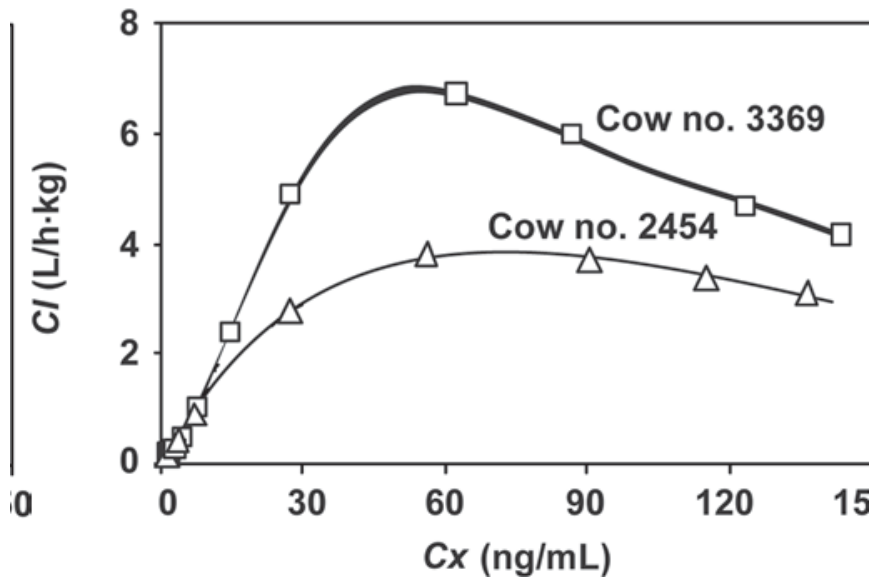

Figure 5. Elimination constant $(\mathrm{Kel})$, elimination half-life $\left(t_{1 / 2}\right)$, and clearance rate $(C l)$ as a function of plasma progesterone concentration $(C x)$ from model 3 for the highest $(\square)$ and lowest $(\Delta)$ milkproducing cows.

pharmacokinetic behaviors was left open because these are ubiquitous features of systems involving highly lipophilic drugs. After nonlinear regression analysis, the 2-coupled first-order kinetics yielded more accurate data fitting than did the saturated Michaelis-Menten 
Table 4. Comparison between the regression line slope $(B)$ and the parameter model $(V m / V x)$ values for each $\operatorname{cow}^{1}$

\begin{tabular}{lcccc}
\hline Group & Cow no. & $\begin{array}{c}B \\
(\mathrm{ng} / \mathrm{mL} \text { per } \min )\end{array}$ & $\begin{array}{c}V m / V x \\
(\mathrm{ng} / \mathrm{mL} \text { per } \min )\end{array}$ & $\begin{array}{c}C o^{h} / K^{h} \\
\text { (dimensionless) }\end{array}$ \\
\hline A $(\mathrm{n}=5)$ & 3369 & 11.51 & 11.96 & $\gg 1$ \\
& 4237 & 21.40 & 30.08 & $\gg 1$ \\
& 4067 & 14.32 & 19.30 & $\gg 1$ \\
$\mathrm{~B}(\mathrm{n}=5)$ & 4548 & 27.19 & 32.07 & $\gg 1$ \\
& 3668 & 17.44 & 24.24 & $\gg 1$ \\
& 4450 & 12.80 & 10.94 & $\gg 1$ \\
& 2896 & 5.40 & 1.40 & $\gg 1$ \\
& 3690 & 30.84 & 10.65 & $\gg 1$ \\
\hline
\end{tabular}

${ }^{1} \mathrm{Co}=$ coordinate-intercept of the regression line on initial times of plasma progesterone concentration curve; $\mathrm{Co}^{h} / K^{h}>>1$ corroborate that the system is saturated at $C o$ and hormone is depurated to constant rate, according to the Hill equation.

kinetics. This fact can provide a valid argument in favor of the widespread use of the former model to describe progesterone profiles in various species, including camel (Al-Busadah and Homeida, 2004), monkey (Anand Kumar et al., 1982), sheep (Contreras-Solis et al., 2008), rabbit (Corbo et al., 1988), and human (Little et al., 1966). Our experimental observations and kinetic modeling, however, support the occurrence of a saturation metabolism of progesterone following a sigmoidal behavior, which is better described by the Hill equation than by the 2-exponent decay. This newer finding indicates the presence of cooperative binding interactions of progesterone with transport protein(s) or metabolizing enzyme(s). The intriguing modeling results reinforce the expectation that the effects can be attributed to cooperative progesterone-binding sites in the enzyme(s) active site and support the validity of the suggested rate-determining step, as discussed below.

It is well known that cytochrome P450 enzymes mediate the oxidative biotransformation of lipophilic compounds in, among other organs, the liver, kidney, brain, ovary and adrenal (Meyer et al., 2002; Plant, 2007; Pegolo et al., 2008). The cytochrome P450 isoforms CYP3A4, CYP2D6, CYP2C9, CYP2C19,
CYP2E1, and CYP1A2 are responsible for the oxidative metabolism of more than $90 \%$ of marketed drugs (Halkier, 1996); principally, CYP3A4 metabolizes more drug molecules than all other isoforms combined (Guengerich, 1997). In humans, CYP3A4 exhibits homotropic and heterotropic cooperativity toward several substrates such as aflatoxin B1, steroids (Schwab et al., 1988), carbamazepine, amitriptyline, diazepam, and its derivates (Harlow and Halpert, 1997; Ueng et al., 1997; Shou et al., 1999) and progesterone (Harlow and Halpert, 1998). An allosteric mechanism is usually invoked to explain the cooperativity. Less is known in bovines, but progesterone metabolism in other mammals is mainly mediated by the CYP2C and CYP3A subfamilies (Murray, 1992; Guengerich, 1997; Lemley et al., 2008). Most P450-mediated reactions follow simple Michaelis-Menten (hyperbolic) kinetics. Many of them, however, exhibit non-Michaelis-Menten kinetics, possibly resulting from an allosteric or cooperative effect that commonly yields sigmoidal saturation curves (Shou et al., 1999). Concurrently, our kinetic analysis revealed a sigmoidal pattern of the progesterone metabolism rate versus plasma progesterone concentration in lactating Holstein cows. Indeed, after kinetic

Table 5. Progesterone concentration and dosage for the maximum $\mathrm{Kel}$ and $\mathrm{Cl}$, and minimum $t_{1 / 2}$ values [mean $(\mathrm{SD})]^{1}$

\begin{tabular}{lcccccc}
\hline Group & $C x(\mathrm{ng} / \mathrm{mL})$ & Dose $(\mathrm{mg} / \mathrm{kg})$ & $K e l \max (/ \mathrm{h})$ & $t_{1 / 2}(\mathrm{~h})$ & $\begin{array}{c}C l \max \\
(\mathrm{L} / \mathrm{h})\end{array}$ & $\begin{array}{c}C l \text { max } / \text { weight } \\
(\mathrm{L} / \mathrm{h} \text { per kg) }\end{array}$ \\
\hline $\mathrm{A}(\mathrm{n}=5)$ & 99.28 & $0.07^{\mathrm{a}}$ & $8.40^{\mathrm{b}}$ & $0.08^{\mathrm{b}}$ & $3,567^{\mathrm{b}}$ & $5.80^{\mathrm{b}}$ \\
& $(35.86)$ & $(0.02)$ & $(1.69)$ & $(0.01)$ & $(775)$ & $(0.94)$ \\
$\mathrm{B}(\mathrm{n}=5)$ & 73.71 & $0.06^{\mathrm{a}}$ & $4.35^{\mathrm{b}}$ & $0.17^{\mathrm{b}}$ & $2,231^{\mathrm{b}}$ & $3.53^{\mathrm{b}}$ \\
& $(15.99)$ & $(0.01)$ & $(1.53)$ & $(0.04)$ & $(635)$ & $(1.18)$ \\
\hline
\end{tabular}

${ }^{a}$ Means between groups were equal $(P>0.05)$.

${ }^{\mathrm{b}}$ Means between groups were different $(P<0.05)$.

${ }^{1} \mathrm{Cx}=$ progesterone concentration; $\mathrm{Kel}=$ elimination constant; $t_{1 / 2}=$ elimination half-life; $\mathrm{Cl}=$ clearance rate. 
modeling using multi-parametric nonlinear regression analysis and goodness-of-fit criteria, the Hill (sigmoidal) equation has proved to be better than both the widely accepted 2-coupled first-order (bi-exponential) and Michaelis-Menten (hyperbolic) kinetic equations. To our knowledge, there are no published models using the Hill equation to describe the metabolism rate of progesterone in dairy cattle. New insights arise from this finding especially those related to some mechanistic aspects of the enzyme-mediated reaction step and to the pharmacokinetic performance of progesterone in cows with different daily milk yields.

The values [mean (SD)] of the Hill coefficient $h$ were found to be $2.38(0.23)$ and $2.08(0.41)$ for A and B groups of cows, respectively. These values are in very close agreement with those reported in pharmacokinetic modeling studies involving other mammals, humans included (Harlow and Halpert, 1998). The Hill coefficient is generally used to estimate the number of ligand molecules that are required for binding to a receptor to cause a functional effect, but the Hill coefficient accurately estimates the number of binding sites only under specific conditions of marked cooperativity. A kinetic mechanism involving 2 cooperative progesterone-binding sites in a cytochrome $\mathrm{P} 450$ active site could be hypothesized. The Hill coefficient, however, must be thought of as an interaction coefficient, reflecting the extent of cooperativity among multiple ligand binding sites. In the present study, the effect was observed to be definitely positive; that is, the binding of the first progesterone molecule increased the affinity and reaction rate of the other vacant sites for progesterone, resulting in so-called cooperative binding. Positive cooperativity makes enzymes much more sensitive to plasma progesterone concentration, and their activities can undergo significant changes in a narrow range of hormone concentration.

We found that the sigmoidal response renders 1) a progesterone metabolism rate slower than that expected for normal kinetics at low plasma progesterone concentrations, specifically in the range of 1 to $15 \mathrm{ng} / \mathrm{mL} ; 2$ ) a much more sensitive progesterone metabolism rate than linear or hyperbolic kinetics for plasma progesterone concentration variations ranging from 15 to 120 $\mathrm{ng} / \mathrm{mL}$ - more concisely, an enhancing rate in the range of 15 to $60 \mathrm{ng} / \mathrm{mL}$ followed by a decreasing rate in the range of 60 to $120 \mathrm{ng} / \mathrm{mL}$; and 3) a saturated metabolism for plasma progesterone concentrations $>120 \mathrm{ng} /$ $\mathrm{mL}$. The described effects are greater for group A compared with group B animals. The values of the kinetic parameters $K$ and $h$ do not change with the daily milk yields, but the intrinsic maximum rate of metabolism $V m$ for group A (high-producing dairy cows) is twice that of group B (low-producing dairy cows). From this recognition, we cannot rule out the possibility that the relative increased progesterone metabolism and, thereby, the greater metabolic clearance rate in highproducing dairy cows may further result from a relative major abundance of CYP2C and CYP3A, not only from an increase in blood flow as a consequence of a greater DMI as usually reported (Sangsritavong et al., 2002; Vasconcelos et al., 2003; Wiltbank et al., 2006). Our argument of an increased expression of CYP2C and CYP3A is based on the following additional findings. First, the metabolic demands of high-producing dairy cows result in a greater negative energy balance, during which blood concentrations of insulin are reduced (Butler, 2000, 2003; Shrestha et al., 2004). Second, recent observations have shown that the metabolism rate of progesterone can be dramatically reduced by decreasing the relative abundance of hepatic CYP2C and CYP3A in dairy cows, as experimentally corroborated in animals infused with insulin (Lemley et al., 2008). From these outcomes, a greater expression of CYP2C and CYP3A could be expected in high-producing dairy cows, as we ascertained from the $V m$ values obtained in the present kinetic study. We recognize, however, that the validity of this assertion remains to be corroborated by lacking essential information on determining the abundance of these enzymes and insulin concentrations in the current study.

The sigmoidal kinetic pattern determines a performance of the $\mathrm{Kel}, t_{1 / 2}$, and $\mathrm{Cl}$ parameters strongly dependent on the concentration of progesterone in plasma. This feature could contribute, at least in part, to the variability seen in the values reported in the literature. Indeed, the present study shows that the values of these pharmacokinetic parameters could differ markedly within the range of low to intermediate plasma progesterone concentrations, which are of interest in dairy cattle reproductive management programs. The maximum values of $\mathrm{Kel}$ and $\mathrm{Cl}$, and thereby the minimum $t_{1 / 2}$, were found for plasma progesterone concentrations ranging from 70 to $100 \mathrm{ng} / \mathrm{mL}$; that is, for a progesterone dose of 0.06 to $0.07 \mathrm{mg} / \mathrm{kg}$ for both groups of cows. Extreme values of these parameters can be found in Table 5. Our attempts to compare our data with those reported in the literature were generally futile in terms of finding a complete data set (i.e., pharmacokinetic parameters and plasma progesterone concentrations). Values of metabolic clearance rates reported for unfed groups of nonlactating and lactating cows of 2.61 and $4.26 \mathrm{~L} / \mathrm{h}$ per $\mathrm{kg}$, respectively, are exceptionally similar to our results for high- and low-producing dairy cows that remained unfed during assays (Sangsritavong et al., 2002). We note that to provide a comparison framework, the specification of the progesterone concentration in plasma is mandatory. 
The sigmoidal kinetic pattern has practical implications. At intermediate progesterone concentrations, small changes in the progesterone concentration produce great variations of the metabolism rate. At low progesterone concentrations, on the contrary, small variations of the metabolism rate produce significant changes in the progesterone concentration. This explains why high progesterone concentrations in blood are rapidly depleted until concentrations of about 10 to $15 \mathrm{ng} / \mathrm{mL}$ are achieved, as observed after injection of progesterone solutions; and why low progesterone concentrations in blood are difficult to maintain within a concentration range of 2 to $10 \mathrm{ng} / \mathrm{mL}$, as observed during intravaginal administration of progesterone released from controlled internal drug-release devices.

\section{CONCLUSIONS}

Some insights into progesterone metabolism were discussed from a kinetic modeling viewpoint. After nonlinear regression of the kinetic parameters and statistical analysis of the data-fitting capability of 3 proposed kinetic models, a simple one-compartment model based on the Hill equation proved to be most adequate for describing the pharmacokinetics within a plasma progesterone concentration ranging from linear to saturated metabolism. To our knowledge, there are no published models using the Hill (sigmoidal) equation to describe the metabolism rate of progesterone in dairy cattle. The proposed kinetic model supports an enzyme-catalyzed metabolism of progesterone involving cooperative substrate-binding sites, resulting from allosteric effects that yield a sigmoidal saturation rate curve. A remarkable quantitative agreement was found between the Hill coefficient value and that reported in pharmacokinetic studies involving CYP3A-mediated reactions in other mammals, including humans. Kinetic parameters were estimated for 2 groups of lactating Holstein cows with different daily milk yields. Positive cooperativity makes enzymes much more sensitive to plasma progesterone concentration, and their activities can undergo significant changes over a narrow range of hormone concentration. Therefore, the performance of pharmacokinetic parameters, such as the elimination constant, elimination half-life, and clearance rate, was found to be highly dependent on the plasma progesterone concentration in lactating Holstein cows.

\section{ACKNOWLEDGMENTS}

The authors express their gratitude to Agencia Nacional de Promoción Científica y Tecnológica (ANPCYT, Buenos Aires, Argentina), Consejo Nacional de Investigaciones Científicas y Técnicas (CONICET, Buenos
Aires, Argentina), and Universidad Nacional del Litoral (UNL, Santa Fe) of Argentina, for the financial support granted to this contribution.

\section{REFERENCES}

Al-Busadah, K. A., and A. M. Homeida. 2004. Pharmacokinetics of progesterone in dromedary camels (Camelus dromedarim). Res. Vet. Sci. $77: 245-247$.

Anand Kumar, T. C., G. F. David, A. Sankaranarayanan, V. Puri, and K. R. Sundram. 1982. Pharmacokinetics of progesterone after its administration to ovariectomized rhesus monkeys by injection, infusion, or nasal spraying. Proc. Natl. Acad. Sci. USA 79:41854189.

Bedford, C. A., F. A. Harrison, and R. B. Heap. 1974. Splanchnic, uterine, ovarian and adrenal uptake of progesterone and 20 alphadihydroprogesterone in the pregnant and non-pregnant sheep. J. Endocrinol. 62:277-290.

Brankin, R. W., I. Gladwell, and L. F. Shampine. 1991. RKSUITE: A Suite of Runge Kutta Codes for the Initial Value Problem for ODES. Softreport 91-1, Mathematics Department, Southern Methodist University, Dallas, TX.

Butler, W. R. 2000. Nutritional interactions with reproductive performance in dairy cattle. Anim. Reprod. Sci. 60-61:449-457.

Butler, W. R. 2003. Energy balance relationships with follicular development, ovulation and fertility in postpartum dairy cows. Livest. Prod. Sci. 83:211-218.

Colazo, M. G., J. P. Kastelic, H. Davis, M. D. Rutledge, M. F. Martinez, J. A. Small, and R. J. Mapletoft. 2008. Effects of plasma progesterone concentrations on LH release and ovulation in beef cattle given GnRH. Domest. Anim. Endocrinol. 34:109-117.

Contreras-Solis, I., A. Gomez-Brunet, T. Encinas, A. Gonzalez-Bulnes, J. Santiago-Moreno, and A. Lopez-Sebastian. 2008. Influence of vehicle on kinetics of exogenous progesterone administered either by subcutaneous and intramuscular routes to sheep. Res. Vet. Sci. 85:162-165.

Corbo, D. C., Y. C. Huang, and Y. W. Chien. 1988. Nasal delivery of progestational steroids in ovariectomized rabbits. I. Progesterone - comparison of pharmacokinetics with intravenous and oral administration. Int. J. Pharm. 46:133-140.

Freetly, H. C., and C. L. Ferrell. 1994. Net uptakes of oestradiol-173 and progesterone across the portal-drained viscera and the liver of ewes. J. Endocrinol. 141:353-358.

Froment, G. F., and K. B. Bischoff. 1990. Chemical Reactor Analysis and Design. 2nd ed. John Wiley \& Sons, New York, NY.

Froment, G. F., and L. H. Hosten. 1981. Catalysis Science and Technology. Vol. 2. Springer-Verlag, Berlin, Germany.

Gomes, W. R., and R. E. Erb. 1965. Progesterone in bovine reproduction: A review. J. Dairy Sci. 48:314-330.

Guengerich, F. P. 1997. Comparisons of catalytic selectivity of cytochrome P450 subfamily enzymes from different species. Chem. Biol. Interact. 106:161-182.

Halkier, B. A. 1996. Catalytic reactivities and structure/function relationships of cytochrome P450 enzymes. Phytochemistry 43:121.

Hamudikuwanda, H., G. Gallo, E. Block, and B. R. Downey. 1996. Adipose tissue progesterone concentrations in dairy cows during late pregnancy and early lactation. Anim. Reprod. Sci. 43:1523.

Harlow, G. R., and J. R. Halpert. 1997. Alanine-scanning mutagenesis of a putative substrate recognition site in human cytochrome $\mathrm{P} 450$ 3A4. Role of residues 210 and 211 in flavonoid activation and substrate specificity. J. Biol. Chem. 272:5396-5402.

Harlow, G. R., and J. R. Halpert. 1998. Analysis of human cytochrome P450 3A4 cooperativity: Construction and characterization of a site-directed mutant that displays hyperbolic steroid hydroxylation kinetics. Biochemistry 95:6636-6641.

Lemley, C. O., S. T. Butler, W. R. Butler, and M. E. Wilson. 2008. Insulin alters hepatic progesterone catabolic enzymes cytochrome P450 2C and 3A in dairy cows. J. Dairy Sci. 91:641-645. 
Little, B., J. F. Tait, S. A. Tait, and F. Erlenmeyer. 1966. The metabolic clearance rate of progesterone in males and ovariectomized females. J. Clin. Invest. 45:901-912.

Marquardt, D. W. 1963. An algorithm for least squares estimation of nonlinear parameters. J. Soc. Ind. Appl. Math. 11:431-441.

Mehvar, R. 2001. Principles of nonlinear pharmacokinetics. Am. J. Pharm. Educ. 65:178-184.

Meyer, R. P., M. Podvinec, and U. A. Meyer. 2002. Cytochrome P450 CYP1A1 accumulates in the cytosol of kidney and brain and is activated by heme. Mol. Pharmacol. 62:1061-1067.

Miller, W. R., R. Williams, O. W. Pipes, and C. W. Turner. 1963. Conjugation, distribution, and biological half-life ( $t$ 1/2) of radioactive progesterone in plasma and red cells of bovine blood. J. Dairy Sci. 46:1402-1404.

Murray, M. 1992. Participation of a cytochrome P450 enzyme from the 2C subfamily in progesterone 21-hydroxylation in sheep liver. J. Steroid Biochem. Mol. Biol. 43:591-593.

Parr, R. A., I. F. Davis, M. A. Miles, and T. J. Squires. 1993. Liver blood flow and metabolic clearance rate of progesterone in sheep. Res. Vet. Sci. 55:311-316.

Pegolo, S., R. Merlanti, M. Giantin, M. Dacasto, C. Montesissa, and F. Capolongo. 2008. High performance liquid chromatography determination of cytochrome $\mathrm{P} 4501 \mathrm{~A}$ and $2 \mathrm{C}$ activities in bovine liver microsomes. Vet. J. doi:10.1016/j.tvjl.2008.08.006

Plant, N. 2007. The human cytochrome P450 sub-family: Transcriptional regulation, inter-individual variation and interaction networks. Biochim. Biophys. Acta 1770:478-488.

Rabiee, A. R., D. Dalley, J. M. Borman, K. L. Macmillan, and F. Schwarzenberger. 2002. Progesterone clearance rate in lactating dairy cows with two levels of dry matter and metabolisable energy intakes. Anim. Reprod. Sci. 72:11-25.

Rabiee, A. R., K. L. Macmillan, and F. Schwarzenberger. 2001a. Progesterone metabolism in ovariectomised non-lactating Holstein-
Friesian cows treated with progesterone with two levels of feed intake. Anim. Reprod. Sci. 66:35-46.

Rabiee, A. R., K. L. Macmillan, and F. Schwarzenberger. 2001b. The effect of level of feed intake on progesterone clearance rate by measuring faecal progesterone metabolites in grazing dairy cows. Anim. Reprod. Sci. 67:205-214.

Sangsritavong, S., D. K. Combs, R. Sartori, L. E. Armentano, and M. C. Wiltbank. 2002. High feed intake increases liver blood flow and metabolism of progesterone and estradiol-17 $\beta$ in dairy cattle. J. Dairy Sci. 85:2831-2842.

Schwab, G. E., J. L. Raucy, and E. F. Johnson. 1988. Modulation of rabbit and human hepatic cytochrome P-450-catalyzed steroid hydroxylations by alpha-naphthoflavone. Mol. Pharmacol. 33:493-499.

Shou, M., Q. Mei, J. Michael, W. Ettore, R. Dai, T. A. Baillie, and T. H. Rushmore. 1999. Sigmoidal kinetic model for two co-operative substrate-binding sites in a cytochrome P450 3A4 active site: An example of the metabolism of diazepam and its derivatives. Biochem. J. 340:845-853.

Shrestha, H. K., T. Nakao, T. Higaki, T. Suzuki, and M. Akita. 2004 Resumption of postpartum ovarian cyclicity in high-producing Holstein cows. Theriogenology 61:637-649.

Ueng, Y. F., T. Kuwabara, Y. J. Chun, and F. P. Guengerich. 1997. Cooperativity in oxidations catalyzed by cytochrome P450 3A4. Biochemistry 36:370-381.

Vasconcelos, J. L. M., S. Sangsritavong, S. J. Tsai, and M. C. Wiltbank. 2003. Acute reduction in serum progesterone concentrations after feed intake in dairy cows. Theriogenology 60:795-807.

Williams, W. F. 1962. Excretion of progesterone and its metabolites in milk, urine, and feces. J. Dairy Sci. 45:1541-1542.

Wiltbank, M., H. Lopez, R. Sartori, S. Sangsritavong, and A. Gümen. 2006. Changes in reproductive physiology of lactating dairy cows due to elevated steroid metabolism. Theriogenology 65:17-29. 